\title{
$\underline{\text { La jeunesse canadienne et le déficit électoral }}$
}

\author{
Gilbert Desgrosseilliers
}

Collège militaire royal du Canada

Gilbert Desgrosseilliers est un Franco-ontarien qui réside actuellement à Kingston, Ontario. Il est un étudiant de 4 e année au Collège militaire royal du Canada, qui termine une spécialisation en politique. En raison de son dévouement aux études et de ses nombreux talents, il a participéà plusieurs conférences politiques telles que la Simulation internationale canadienne des Nations Unies à Ottawa en 2006, la Conférence étudiante sur la politique étrangère américaine (SCUSA) à West Point en 2006, la Conférence internationale des Élèves-officiers à l’Académie de défense nationale au J apon en 2007 et la Compétition internationale sur le droit des conflits armés à Sanremo en Italie en 2007. 
$\underline{\text { Introduction }}$

La chute du mur de Berlin en 1989 a marqué la victoire du libéralisme démocratique au détriment de l'idéologie communiste. Les conditions étaient donc propices au fleurissement mondial des principes et des valeurs démocratiques. Malgré son hégémonie idéologique incontestée, la démocratie a connu depuis 1989 certains échecs et plusieurs difficultés. Tout d'abord, l'exportation de la démocratie a fait débâcle en Afghanistan et en Iraq, où les gouvernements en place ne réussissent pas à consolider leur emprise sur leur pays. De plus, en Somalie, malgré l'existence d'un « gouvernement élu », le pays est, depuis dix ans, profondément ancré dans l'anarchie et tristement à la merci des seigneurs de guerre guerroyants.

Toutefois, on ne peut pas se permettre d'imaginer que la démocratie ne connaisse des difficultés que dans les pays en voie de développement ou dans les États ratés ( « failed states »). Au contraire, partout où elle est l'idéologie dominante, la démocratie est en état de légère crise. Cette crise se caractérise par un déficit électoral, c'est-à-dire une baisse importante de la proportion de la population qui se présente au jour du scrutin afin d'exercer sa souveraineté en choisissant ses représentants auprès du gouvernement. Mais, ce déficit est-il universel ou se manifeste-t-il seulement au sein d'une subdivision spécifique de la population ? En effet, c'est parmi les jeunes qu'on aperçoit le manque de participation le plus important. Plus spécifiquement, c'est au sein de la tranche d'âge qui se situe de dix-huit à vingt-quatre ans que l'on rencontre le plus de difficultéà motiver les électeurs à adopter un sentiment civique. ${ }^{1}$

$\mathrm{Au}$ Canada, comme dans presque tous les autres pays membres du G8, si le parti « non au vote! » avait existé lors des élections en 2004, on dit qu'il aurait forméle gouvernement ${ }^{2}$. De 1975 à 1980, on estime qu'en moyenne, $75 \%$ des électeurs ont voté 3 aux élections fédérales tandis qu'aux élections fédérales de 2004, seulement 60,9\% ont exercé leur droit civique. ${ }^{4}$ Le plus grand facteur qui influence ce faible taux de participation est l'âge. Tandis que les Canadiens âgés de 25 ans

\footnotetext{
${ }^{1}$ Jon H. Pammett, « Youth in the Electoral Process », Electoral Insight, janvier 2001, p. 15

${ }^{2}$ André Turcotte, « Different Strokes : Why Young Canadians Don't Vote », Electoral Insight, janvier 2005, p.1

${ }^{3}$ Wayne Brown, « Electoral Facts », Electoral Insight, janvier 2001, p. 30

${ }^{4}$ Elisabeth Gidengil, André Blais, Joanna Everitt, Patrick Fournier et Neil Nevitte, « Missing the Message : Young Adults and the Election Issues », Electoral Insight, janvier 2005, p. 2
} 
et plus votent à un taux acceptable, seulement $25 \%$ des jeunes se sont présentés au dernier scrutin. ${ }^{5}$

Inutile de dire que ces faits suscitent une certaine inquiétude parmi les administrateurs publics et les politiciens qui craignent de voir cette tendance s'accentuer. À la lumière de ces faits troublants, il est nécessaire de cibler les causes de ce phénomène ainsi que les souscatégories de jeunes Canadiens qui témoignent de ce phénomène afin d'élaborer des plans qui pourraient corriger cette situation non souhaitable.

\section{Causes du déficit électoral}

Les médias et les critiques ont élaboré certaines hypothèses afin d'expliquer le déficit électoral parmi les jeunes. Nous allons aborder chacune de ces hypothèses afin de juger si elles sont vraies ou non.

\subsection{Cycle de la vie et les différences générationnelles}

L'hypothèse la plus répandue est celle du cycle de la vie (« life-cycle») qui est axée sur la croyance que les jeunes Canadiens

\footnotetext{
${ }^{5}$ « Election Basics », Élections Canada, consulté le 06 mars 2006 dans :

http://www.elections.ca/content youth.asp?section=yth\&dir=b as\&document=index\&lang=e
}

n'accordent pas d'importance au processus électoral et politique en raison de leur mode de vie très dynamique. On suppose qu'ils cherchent tout d'abord à se trouver une place dans le monde. Ils doivent donc employer la quasi-totalité de leur énergie et de leur temps pour se chercher un emploi, compléter leurs études et entretenir des relations soient amicales ou amoureuses. ${ }^{6}$ Afin de combler leurs besoins éducationnels, professionnels et relationnels, ils sont donc souvent obligés de se déplacer. Par conséquent, leur mode de vie est très instable et ne favorise pas une participation active à la politique, aux élections et à la vie civique, une participation qui requiert beaucoup de lecture, d'énergie et de temps.

\section{À la lumière de ces faits, on devrait} supposer que ce manque de temps est unique à la jeunesse canadienne d'aujourd'hui, ce qui est faux. La jeunesse d'hier a aussi eu à se déplacer pour aller à l'université, trouver des emplois et entretenir des relations sérieuses. Par contre, aujourd'hui, les jeunes ont même l'avantage de se retrouver dans l'ère de l'information : Ils peuvent avoir accès aux informations politiques par l'intermédiaire de la télévision, des ordinateurs et surtout de l'Internet, ce qui n'était pas toujours possible avant 1980. Ainsi,

\footnotetext{
${ }^{6}$ Jon H. Pammett, Loc. Cit., p. 15
} 
la jeunesse d'aujourd'hui se trouve dans une situation qui favorise la participation politique et le civisme. Alors pourquoi ne vote-elle pas?

Le problème n'est donc pas situationnel, puisque les jeunes ont toujours eu à se déplacer et n'ont jamais eu le luxe de jouir de beaucoup de loisirs dans lequel il a été possible de s'intéresser à la politique et au civisme. Est-ce donc une question d'éducation?

\section{2 Éducation}

La différence la plus marquante est celle de l'éducation. J amais dans l'histoire du Canada a-t-on vu tant de jeunes Canadiens terminer le secondaire, mais surtout, compléter ou poursuivre des études universitaires.

L'éducation est, avec l'âge, un des deux facteurs qui influencent le plus la participation électorale chez un individu. ${ }^{7}$ L'impression générale est que l'influence qu'exerce l'éducation sur le vote est négative. En d'autres mots, on croit que plus on est éduqué, moins on s'intéresse à la politique : « Because they are highly educated, they aspire to something more meaningful than casting a ballot once in a

\footnotetext{
${ }^{7}$ « Youth Electoral Participation - Survey and Analysis of Canadian Trends (October 2003) », Élections Canada, consulté le 06 mars 2006 dans :

http://www.elections.ca/content.asp?section=med\&document= survey\&dir=eveyou/forum\&l...
}

while. ${ }^{8}$ Les faits nous montrent que cette impression est fausse et que l'éducation a un effet positif sur la participation électorale. Ainsi, les universitaires votent en plus grand nombre que ceux qui n'ont pas terminé le secondaire ou se sont intégrés à la main d'œuvre :

Education remains one of the best predictors of turnout because it provides the cognitive skills needed to cope with the complexities of politics and because it seems to foster norms of civic engagement [...] The 2000 Canadian Election Study reveals that turnout in the youngest generation was almost 50 points higher among university graduates than it was among those who left school without a high school diploma. ${ }^{9}$

On peut donc penser que puisqu'il y a actuellement plus de jeunes à l'université qu'il y en avait il y a trente ans, il y aurait donc un plus haut taux de participation aux élections. Malgré la croissance du nombre d'universitaires, ce nombre est toujours bien inférieur à celui des jeunes qui ne sont pas inscrits à l'université : Sur une population totale de 23901360 Canadiens âgés de quinze ans et plus en 2001, il n'y a que 6173225 qui ont complétél'université ou qui poursuivent

\footnotetext{
${ }^{8}$ Elisabeth Gidengil, André Blais, Neil Nevitte, Richard Nadeau, " Turned Off or Tuned Out ? : Youth Participation in Politics », Electoral Insight, juillet 2003, p. 10

${ }^{9}$ Elisabeth Gidengil, André Blais, Neil Nevitte, Richard Nadeau, Loc. Cit., p. 10
} 
toujours leurs études ${ }^{10}$. Toutefois, il faut se mettre en garde de croire que ces 6173225

Canadiens universitaires sont tous des jeunes. Ce nombre inclus égalent les Canadiens âgés de vingt-quatre ans et plus qui sont actuellement inscrits à l'université où qui ont déjà complété leurs études. Bref, malgré la forte participation électorale de la part des universitaires, ils sont trop peu pour modifier les tendances électorales générales qui sont davantage influencées par les jeunes non-universitaires qui sont bien plus nombreux.

\subsection{Cycle Motivation-Connaissances}

Il est vrai que l'éducation fournit aux jeunes les facultés cognitives nécessaires pour comprendre et participer à la politique, mais ces moyens ne servent à rien si les jeunes ne sont pas sensibilisés au processus électoral. En d'autres mots, comment un jeune peut-il participer à la politique s'il ne connaît rien à ce sujet? De plus, comment motiver ce même jeune à exercer son devoir civique s'il ignore la nature même de ce devoir ? En fait, la motivation et les connaissances civiques/politiques sont au coeur même du problème en question.

\footnotetext{
10 « Population 15 Years and Over by Highest Level of Schooling (1981-2001 Censuses) », Statistique Canada, consulté le 06 mars 2006 dans :

http://www40.statcan.ca/101/cst01/educ45.htm
}

Ainsi, il faut posséder des connaissances politiques afin d'être motivé à pousser ses connaissances dans le domaine politique, mais, il est également vrai qu'il faut de la motivation afin d'aller chercher par soi-même ces connaissances fondamentales puisqu'elles ne sont ni fournies par le système scolaire ni par l'État. Le problème réside donc dans le fait que les connaissances fondamentales ainsi que la motivation manquent aux jeunes Canadiens. En fait, lorsqu'on a demandé à de jeunes à remplir un sondage ayant comme objectif de mettre à l'épreuve leurs connaissances politiques, les résultats n'ont pas été très encourageants :

When they were interviewed right after the 2000 federal election, almost one young Canadian in five was unable to name J ean Chrétien as the leader of the Liberal party [...] Young Canadians knew even less about the party's positions than older Canadians. Only one in four could identify the Alliance as being on the right and even fewer could locate the N.D.P. as being on the left. ${ }^{11}$

\section{Puisqu'on ne peut pas facilement} motiver les jeunes à s'intéresser à la politique, surtout ceux de seize à dix-huit ans, on peut se contenter au moins de leur faire apprendre des connaissances civiques fondamentales. C'est

\footnotetext{
${ }^{11}$ Elisabeth Gidengil, André Blais, Neil Nevitte, Richard Nadeau, Loc. Cit., p. 11
} 
ainsi que le gouvernement provincial progressiste-conservateur de Mike Harris en Ontario a fait une première tentative pour redresser la situation de déficit électoral et d'ignorance politiques/ civiques. Parmi les maintes réformes imposées au système d'éducation, Mike Harris a introduit, en 2000, au programme d'éducation, un nouveau curriculum standardisé dans lequel chaque étudiant en dixième année, âgé de seize ans, doit suivre un cours intitulé civisme (« Civics »). ${ }^{12}$ Ce cours a comme objectif de sensibiliser les jeunes Ontariens à leur devoir en tant que citoyen canadien ainsi qu'à les encourager à participer à la vie civique dans un système libéro-démocratique.

Cette approche, axée sur une éducation obligatoire, semble être plus efficace qu'un système scolaire dans lequel on offre le choix aux étudiants de suivre un cours de civisme ou non. En donnant un cours de civisme nonobligatoire, on n'encouragera que les gens qui ont déjà des connaissances et un intérêt en politique à suivre le cours.

\subsection{2 Éducation civique}

Cependant, il ne faut pas se contenter de transmettre les valeurs civiques quelques

\footnotetext{
${ }^{12}$ Paul Howe, « Electoral Participation and the Knowledge Deficit », Electoral Insight, juillet 2003, p. 22
}

années avant l'âge de dix-huit ans; il vaudrait mieux que les jeunes aient assimilé ces connaissances dès leur entrée au primaire. Ainsi, on peut modeler leur comportement afin de favoriser le civisme.

Toutefois, on ne peut pas s'imaginer que l'on doit enseigner la politique et le civisme aux jeunes de six et de sept ans. Ces derniers n'ont pas encore les aptitudes cognitives nécessaires pour bien saisir les concepts fondamentaux du processus politique et électoral. Néanmoins, on peut toujours les habituer à l'idée d'une campagne électorale et à l'importance du vote. Ainsi, Élections Canada a créé deux trousses de simulation en fonction des groupes d'âge et des niveaux de connaissances différents afin de sensibiliser les jeunes à l'importance des élections :

Elections Canada has produced two election simulation kits to aid in the education process at both junior and senior levels. The junior kit, designed for children between 5 and 10 years old, takes the students through a simulated campaign for class mascot, and then conduct a vote. The senior kit, Canada at the Polls!, takes students through the basic process of holding an election, and can be adapted for whatever election the group wishes to hold. ${ }^{13}$ 
Cette initiative de la part d'Élections Canada a connu, à présent, beaucoup de succès. En effet, deux mille exemplaires de ces trousses de simulation sont distribués aux écoles à travers le Canada chaque année. De surcroît, cette même agence a conçu un cédérom intitulé « Exploring Canada's Electoral System » qui offre un survol du système électoral canadien et dont vingt mille exemplaires ont été distribués à la fois aux écoles et aux parents intéressés. ${ }^{14}$

\subsubsection{Réforme électorale}

Ainsi, à seize ans, soit en dixième année du secondaire en Ontario, les jeunes sont sensibilisés au civisme ainsi qu'au système électoral par l'intermédiaire du cours de civisme ainsi que par les trousses de simulation fournies par Élections Canada. On doit donc se demander si ces efforts sont en vain puisque ces mêmes ne pourront exercer leur droit au vote que deux ans plus tard. Pour plusieurs, l'intérêt à l'égard du processus politique et électoral suscité à l'âge de seize ans sera depuis longtemps éteint à l'âge de dix-huit ans, car il n'y aura pas de suivi pour renforcer et consolider un comportement civique. Par conséquent, le civisme ne deviendra pas une

${ }^{14}$ Jon H. Pammett, Loc. Cit., p. 16 habitude bien ancrée et risquera de disparaître avant l'âge de majorité. ${ }^{15}$

Afin de faire renaître ce sentiment civique, disparu depuis deux ans par manque de renforcement, J ean-Pierre Kingsley, l'Officier en chef d'Élections Canada, a annoncé en 2003 son intention d'envoyer aux jeunes Canadiens, en l'honneur de leur dix-huitième anniversaire, une lettre afin de les féliciter d'avoir accédé au statut de citoyen-électeur et de les encourager à s'inscrire à la liste électorale. ${ }^{16}$

Malheureusement, cette tactique n'a pas porté fruit, si on se fie au nombre de jeunes de dix-huit ans qui ont voté lors des dernières élections. Ce nombre fut quasiment pareil aux élections antérieures.

Il faut donc se demander si une baisse de l'âge de majorité sera efficace pour hausser la participation aux élections des jeunes. En Allemagne, six des seize Länder ont, lors des dix dernières années, effectué des amendements au droit électoral : Ils ont donné

\footnotetext{
${ }^{15}$ En psychologie, ce phénomène se nomme l'extinction. On dit qu'un comportement disparaît si le renforçateur qui encourageait jusqu'alors le comportement est éliminé. James Hunt, Richard N. Osborn et John R. Schermerhorn, Comportement humain et organisation, Éditions du renouveau pédagogique, Saint-Laurent, Québec, 2002, p. 134

${ }_{16}$ Jean-Pierre Kingsley, "Youth Participation in Elections », Electoral Insight, juillet 2005, p.1
} 
aux jeunes Allemands de seize ans le droit au vote, droit qui était autrefois obtenu à l'âge de dix-huit ans. ${ }^{17}$ Ainsi, d'autres pays démocratiques tels que le Royaume-Uni et le Canada considèrent suivre l'exemple de l'Allemagne.

Une telle réforme serait peut-être favorable au Canada si on tient compte du cours de civisme obligatoire donné aux élèves ontariens âgés de seize ans. Ces derniers seront donc plus portés à participer aux élections, puisqu'ils pourraient mettre en pratique leurs connaissances politiques nouvellement acquises au sein du cadre du cours de civisme. De plus, on envisage fournir de l'argent aux écoles secondaires en fonction du nombre d'élèves qui sont inscrits sur la liste électorale ${ }^{18}$. On pourrait donc intégrer cette inscription ainsi que l'acte de vote (lorsqu'il y a une élection) dans le contexte du cours, rendant ainsi la participation électorale obligatoire pour les étudiants suivant le cours de civisme. L'expérience allemande en ce domaine appuie cette suggestion :

At age 16, most young persons still attend school. Civic education classes, which are commonly required before age

\footnotetext{
${ }^{17}$ Wayne Brown, « Rush the Vote », Electoral Insight, juillet 2003, p.47

${ }^{18}$ Keith Archer, « Increasing Youth Voter Registration : Best Practices in Targeting Young Electors », Electoral Insight, juillet 2003, p. 28
}

18, may support the socialization into voting habits [...] The research in the three German states on the turnout level of 16-18 years olds, lends some support to this hypothesis. In North RhineWestphalia, the turnout among 16-21 year olds was slightly below the average for the whole electorate, but clearly higher by about 5 to 8 percent - than among those aged 21-30. Similar results hold for Lower Saxony, where 16-18 year olds vote at a level comparable to 3545 year olds. Finally, a similar conclusion can be drawn for the 1999 local elections in SaxonyAnhalt. 19

Toutefois, de telles réformes ne seront pas profitables sur le plan national tant que toutes les provinces et les territoires canadiens n'auront pas suivi l'exemple de l'Ontario et développé un cours obligatoire de civisme pour les étudiants âgés de seize ans.

\section{Malgré que les preuves empiriques} provenant des six Länder allemands appuient la réforme électorale, le Canada est réticent à suivre une pareille réforme. Cette réforme a été rejetée à deux reprises, la première fois en 1990 par une Commission royale portant sur les réformes électorales et le financement des partis (la Commission Lortie) et une seconde

\footnotetext{
${ }^{19}$ Kees Aarts et Charlotte van Hees, « Lowering the Voting Age : European Debates and Experiences », Electoral Insight, juillet 2003, p. 45
} 
fois en 2003 par une commission provinciale québécoise portant sur les réformes des institutions démocratiques. Cependant, le mouvement pro-réforme semble reprendre son souffle au Québec où le Parti Québécois a introduit la réforme électorale dans sa plateforme électorale en 2003. ${ }^{20}$

\subsection{Devoir civique}

La raison principale pour laquelle on doit offrir une éducation civique obligatoire aux jeunes est attribuable au fait que l'esprit civique leur manque. Le civisme ne manquait pas aux jeunes de l'après guerre puisqu'ils avaient développé une prédilection pour les valeurs démocratiques. Au cours des années 1936-1945, la jeunesse canadienne s'était sacrifiée afin de faire valoir les intérêts nationaux et se protéger de la tyrannie. En fait, plusieurs ont même épaulé un fusil sous le drapeau de la brigade Mackenzie-Papineau pour venir à la défense de la démocratie lors de la Guerre civile espagnole de 1936 à 1939. Et encore en 1939, d'autres se sont, par milliers (sauf au Québec), portés volontaires pour lutter contre le fascisme de Mussolini et le nazisme de Hitler.

\footnotetext{
${ }^{20}$ Raymond Hudon et Bernard Fournier, « How Old Is Old Enough to Vote? : Youth Participation in Society », Electoral Insight, juillet 2003, p. 36
}

Conséquemment, les jeunes, qui ont aidé à protéger par leur souffrance, leur misère et leur vie les valeurs démocratiques ont donc glorifié ces dernières.

Une de ces valeurs, on dirait même la valeur fondamentale, est celle du suffrage. Ce n'était donc aucunement une surprise lorsque les Canadiens se sont rués aux urnes lors des élections qui ont suivi la guerre de 1939-1945. Il est à noter que le plus haut taux de participation électorale dans l'histoire canadienne a été lors de l'élection de 1958 au cours de laquelle $79,4 \%$ des électeurs ont voté pour un gouvernement progressisteconservateur sous la direction de J ohn Diefenbaker ${ }^{21}$.

Aujourd'hui, le souvenir de ces sacrifices est bien distant dans la mémoire collective du peuple canadien et nejoue donc pas un grand rôle à façonner un comportement civique au sein de la population chez laquelle les souvenirs de la guerre sont très faibles.

Un autre facteur qui semble avoir contribué à la formation d'une conscience civique chez la génération des « baby boomers » est la religion : « Religiosity may help to develop a sense of duty to vote and thus

\footnotetext{
${ }^{21}$ Wayne Brown, Loc. Cit., p. 30
} 
increase the likelihood of turning out at election time. ${ }^{22}$ Ainsi, avec la laïcisation de la société canadienne, surtout au Québec, ce sens du devoir est perdu.

\section{Sous-groupes de la jeunesse}

Ainsi, le déficit électoral auprès des jeunes s'explique, de façon générale, par un manque de connaissances, de motivation, d'éducation civique, d'un sens de devoir civique et d'un âge de majorité trop élevé. Il resteà comprendre pourquoi certaines sous-catégories spécifiques de la jeunesse ne votent pas.

\section{$\underline{2.1 \text { Sexe }}$}

Malgré que le suffrage ait été accordé aux femmes en 1917 à la suite de maints débats émotifs et en réponse à une prise de conscience politique des femmes sous l'égide des Suffragettes, les jeunes femmes d'aujourd'hui semblent être un peu moins portées à voter : « Low turnout is greater among those with no post-secondary education, those with low and high family income and to some extent women. »23

\footnotetext{
${ }^{22}$ André Blais, Patrick Fournier, Elisabeth Gidengil, Neil Nevitte et Daniel Rubenson, « Accounting for the Age Gap in Turnout », Acta Politica, 2004, volume 39, p. 410

${ }^{23}$ Brenda O’Neil, « Examining Declining Electoral Turnout Among Canada’s Youth », Electoral Insight, juillet 2003, p. 17
}

Cette tendance ne s'explique pas facilement. Si on se fie à la corrélation positive entre l'éducation universitaire et la participation électorale, on aurait tendance à croire que les femmes participeront davantage que les hommes aux élections puisqu'il y a une surreprésentation de femmes à l'université (57\% des étudiants enrôlés en 2003) et un nombre supérieur de femmes qui étudient les sciences sociales (153 900 femmes contre 107 300 hommes en 2003). ${ }^{24}$ Donc comment expliquer le fait que les femmes votent en nombre inférieur à celui des hommes? Cette constatation met en doute la corrélation positive entre une éducation universitaire et la probabilité de voter. Une seule explication vient à l'esprit. À l'exception de l'ancien Premier ministre progressiste-conservateur Kim Campbell, l'électorat ne voit que rarement des modèles féminins illustres dans la politique fédérale canadienne. Par conséquent, à cause de la sous-représentation féminine dans la politique, les femmes ne s'associent pas facilement aux processus politique et électoral puisqu'il leur manque de bons modèles féminins. Le monde politique leur semble être un monde dominé par les hommes et qui ne

\footnotetext{
24 « University Enrolment by Field of Study », Statistique Canada, consulté le 06 mars 2006 dans : http://www.statcan.ca/Daily/English/030331/d030331b.htm
} 
s'intéresse et ne s'adresse pas aux intérêts des femmes.

\subsection{Immigrants}

Contrairement au léger effet négatif qu'ont les jeunes femmes sur le vote, les nouveaux Canadiens, c'est-à-dire les immigrants nouvellement arrivés, exercent une influence très positive. Le taux de participation électorale de la part des immigrants arrivés au Canada depuis 1990 est supérieur à celui des immigrants arrivés avant cette date. Puisque la majorité des nouveaux immigrants se situe entre l'âge de 15 et 35 ans $(58,2 \%)^{25}$, on peut croire que les nouveaux immigrants (qui sont plus jeunes) votent en plus grand nombre que ceux qui sont arrivées depuis longtemps (qui sont plus âgés). De plus, ces immigrants, citoyens canadiens depuis 1990, votent même à un plus haut taux que les citoyens canadiens de naissance. ${ }^{26}$

Ce phénomène s'explique facilement par le fait que les nouveaux immigrants ont un sens du devoir civique très poussé. Ils se sentent reconnaissants envers leur pays d'accueil et ne

\footnotetext{
25 « The Interprovincial Migration of Immigrants to Canada : Profiling the Movers - A National Overview », Citoyenneté et Immigration Canada, consulté le 06 mars 2006 dans : http://www.cic.gc.ca/english/research/papers/interprovincial/in terprovincial-h.html

${ }^{26}$ André Blais, Patrick Fournier, Elisabeth Gidengil, Neil Nevitte et Daniel Rubenson, Loc. Cit., p. 410
}

cherchent pas à le contrarier en refusant d'adhérer à ses valeurs, dont la plus importante est le suffrage universel.

On peut également justifier pourquoi les immigrants arrivés avant 1990 participent avec un taux plus faible: Pour eux, comme pour les citoyens canadiens de naissance, le sens du devoir civique a perdu son importance puisqu'ils sont établis depuis longtemps et n'ont pas à craindre de contrarier certaines valeurs nationales.

\subsection{Autochtones}

En comparaison à la population canadienne générale, le taux de participation électoral de la communauté autochtone du Canada est très faible. Cette tendance est également vraie, voire même accentuée, pour lesjeunes autochtones.

Les autochtones ne sont aucunement indifférents à la politique; au contraire, ils sont bien intéressés. Leur faible taux de participation électorale n'est donc pas, comme pour les jeunes Canadiens, un reflet de leur désintéressement, mais plutôt l'expression de leur refus d'accepter le sort que leur a réservéle gouvernement fédéral : «A participant said that the foundation of democracy is people choosing their own destiny and that the choice 
not to vote is an exercise of democratic rights. »27 Ainsi, le sens du devoir civique est très fort chez les jeunes autochtones, mais leur loyauté est orientée vers leur communauté et non pas vers la fédération canadienne. Les jeunes autochtones cherchent donc à participer à la politique par l'intermédiaire d'organisations qui se retrouvent hors du cadre traditionnel des politiques canadiennes fédérales et provinciales : « The best way to influence government is to be active within their own organizations; in turn, these organizations can make an impact by lobbying members of Parliament and the governement.» 28

Ces jeunes autochtones font partie d'une communauté qui connaît une forte croissance démographique, surtout au sein des villes telles que Régina. Leur nombre s'accroît tandis que leur influence et leur pouvoir politique n'augmentent guère. Par conséquent, il y a un sentiment de frustration à l'égard des priorités fédérales. Ainsi, les politiciens ne s'intéressent

\footnotetext{
27 « Roundtable on Aboriginal Youth and the Federal Electoral Process - January 17, 2004 - Carleton University - Final

Report - Concluding discussion ", Élections Canada, consulté le 06 mars 2006 dans :

http://www.elections.ca/content_youth.asp?section=yth\&dir=c $\mathrm{al} / \mathrm{rec} / \mathrm{abo} \&$ documents $=$ con\&l...

28 « Roundtable on Aboriginal Youth and the Federal Electoral Process - January 17, 2004 - Carleton University - Final Report - Discussion groups », Élections Canada, consulté le 06 mars 2006 dans :

http://www.elections.ca/content_youth.asp?section=yth\&dir=c al/rec/abo\&documents=dis\&la...
}

pas au vote autochtone puisque les autochtones n'ont pas l'habitude de voter; en revanche, les autochtones ne votent qu'en faible nombre puisque les hommes politiques ne s'intéressent pas à eux. Ce cycle vicieux s'intensifie à chaque élection et risque d'empoisonner les relations autochtones-canadiennes.

Par contre, lorsque c'est dans leur intérêt commun de voter, ils le font en grand nombre et de façon uniforme, c'est-à-dire qu'ils vont presque tous voter de la même façon : « [...] turnouts rates in the reforandums sponsored by the Cree and Inuit in northern Quebec prior to the 1995 referendum on Quebec sovereignty were quite high. »29 autochtone et le gouvernement fédéral ne sont déjà pas très cordiales. Les jeunes autochtones se croient relégués à un statut inférieur à celui du reste de la population canadienne. Les autochtones ont raison de penser ainsi : Ils n'ont reçu le droit de voter qu'en 196030, plusieurs traités n'ont pas été respectés, ils ont souvent été déplacés d'une réserve à une autre,

\footnotetext{
${ }^{29}$ « Roundtable on Aboriginal Youth and the Federal Electoral Process - January 17, 2004 - Carleton University - Final Report - Opening Session », Élections Canada, consulté le 06 mars 2006 dans :

http://www.elections.ca/content_youth.asp?section=yth\&dir=c al/rec/abo\&documents=index...

${ }^{30}$ « Roundtable on Aboriginal Youth and the Federal Electoral Process - January 17, 2004 - Carleton University - Final Report - Discussion groups », Loc. Cit.
} 
ces dernières ont favorisé une condition socioéconomique déplorable par rapport au reste de la population canadienne et ils se sentent sousreprésentés au Parlement canadien. ${ }^{31}$

Tous ces facteurs contribuent à développer une pensée qui suggère que la communauté autochtone ne fait pas partie intégrante de la communauté canadienne : « [...] for some Aboriginal youth, voting in federal elections would be a question of participating within an alien nation. »32 Il y a donc une certaine animosité envers le Canada ; les jeunes autochtones rejettent même parfois la citoyenneté canadienne, car elle est le symbole de leur assujettissement au peuple conquérant : « For some, a non acceptance of Canadian citizenship »33

De surcroît, le faible taux de participation aux élections, qui est fortement influencé par une animosité envers le gouvernement fédéral canadien, est accentué par le fait que les autochtones ne reçoivent pas

\footnotetext{
31 « Roundtable on Aboriginal Youth and the Federal Electoral Process - January 17, 2004 - Carleton University - Final Report - Concluding discussion », Loc. Cit.

32 « Roundtable on Aboriginal Youth and the Federal Electoral Process - January 17, 2004 - Carleton University - Final Report - Presentations on "Aboriginal People and Electoral Participation” », Élections Canada, consulté le 06 mars 2006 dans :

http://www.elections.ca/content_youth.asp?section=yth\&dir=c al/rec/abo\&documents=pre\&l...

33 " Roundtable on Aboriginal Youth and the Federal Electoral Process - January 17, 2004 - Carleton University - Final Report - Discussion groups », Loc. Cit.
}

une éducation adéquate. Les jeunes autochtones n'ont souvent pas reçu un enseignement suffisant pour leur permettre de comprendre le système politique et électoral. De plus, ce manque d'éducation renforce les préjugés envers le gouvernement fédéral ainsi qu'envers les non-autochtones. Quant à l'éducation civique, il y a une certaine ignorance universelle de la politique. ${ }^{34}$

À la lumière de ces faits, le gouvernement fédéral a beaucoup de chemin à faire. Tout d'abord, il doit s'assurer de hausser la qualité du système scolaire des autochtones et de fournir à ces derniers un enseignement civique convenable. Ainsi, les jeunes autochtones pourront reconnaittre leur pouvoir politique potentiel et sauront le développer par la voie électorale. De plus, le gouvernement fédéral doit se forcer à interagir davantage avec la communauté autochtone et de lui accorder une certaine importance afin de ne pas marginaliser politiquement ce peuple qui se sent subjugué. Bref, il faut que les politiciens prennent le premier pas pour mettre un terme au cycle vicieux de l'indifférence réciproque en sollicitant le vote autochtone et en intégrant les intérêts de cette communauté dans leurs plateformes électorales.

\footnotetext{
${ }^{34}$ « Roundtable on Aboriginal Youth and the Federal Electoral Process - January 17, 2004 - Carleton University - Final Report - Discussion groups », Loc. Cit.
} 


\section{$\underline{\text { Conclusion }}$}

La situation dans laquelle se retrouve la jeunesse autochtone par rapport au processus politique et électoral résume bien la question du déficit électoral des jeunes Canadiens.

D'une part, il y a, chez les jeunes, un manque définitif de connaissances politiques et civiques, ce qui ne favorise pas leur compréhension du processus politique. Cette incapacité de comprendre les élections décourage les jeunes d'y participer. De plus, un manque de conscience civique fait en sorte que les jeunes ne reconnaissent pas le besoin et n'apprécient pas l'importance de voter.

D'une autre part, comme c'est le cas pour les femmes et les autochtones, il y a une sous-représentation importante de la jeunesse auprès du gouvernement. Puisqu'il est fort improbable que les jeunes puissent un jour être directement représentés au Parlement canadien, les politiciens doivent s'engager à défendre les intérêts de la jeunesse afin qu'elle ne se sente plus comme une communauté marginalisée et qu'elle croit pouvoir exercer une influence sur les affaires de leur pays. Peut-être donc que les hommes politiques du Canada ont cherché à redresser la situation de déficit électoral par de mauvais moyens. Ils ont 


\section{Bibliographie}

\section{Livre}

HUNT, J ames G.; Osborn, Richard N.; Schermerhorn, J ohn R.. Comportement humain et organisation. Québec, Éditions Renouveau Pédagogique, 2002, 642 p.

\section{Références Internet}

«Election Basics ». Élections Canada. consulté le 06 mars 2006 dans : http:// www.elections.ca/content youth.asp?section=yth\&dir=bas\&document=index\&lang=e

« Marital Satus, 1996 ». The Atlas of Canada. consulté le 06 mars dans : http://atlas.gc.ca/ site/ english/ maps/ peopleandsociety/ family/ family1996/ maritalstatus/ 1

« Population 15 Years and Over by Highest Level of Schooling (1981-2001 Censuses) ». Statistique Canada. consulté le 06 mars 2006 dans : http:// www40.statcan.ca/101/cst01/ educ45.htm

« Roundtable on Aboriginal Youth and the Federal Electoral Process - J anuary 17, 2004 - Carleton University - Final Report - Opening Session ». Élections Canada. consulté le 06 mars 2006 dans : http:// www.elections.ca/ content youth.asp?section=yth\&dir=cal/rec/abo\&documents=index...

« Roundtable on Aboriginal Youth and the Federal Electoral Process - J anuary 17, 2004 - Carleton University - Final Report - Presentations on "Aboriginal People and Electoral Participation" ». Élections Canada. consulté le 06 mars 2006 dans :

http:/ / www.elections.ca/ content_youth.asp?section=yth\&dir=cal/ rec/abo\&documents=pre\&l...

« Roundtable on Aboriginal Youth and the Federal Electoral Process - J anuary 17, 2004 - Carleton University - Final Report - Discussion groups ». Élections Canada. consultéle 06 mars 2006 dans : http:/ / www.elections.ca/ content_youth.asp?section=yth\&dir=cal/ rec/ abo\&documents=dis\&la...

« Roundtable on Aboriginal Youth and the Federal Electoral Process - J anuary 17, 2004 - Carleton University - Final Report - Concluding discussion ». Élections Canada. consulté le 06 mars 2006 dans :

http:/ / www.elections.ca/ content_youth.asp?section=yth\&dir=cal/ rec/abo\&documents=con\&l...

« The Interprovincial Migration of Immigrants to Canada : Profiling the Movers - A National Overview ». Citoyenneté et Immigration Canada. consulté le 06 mars 2006 dans : http:// www.cic.gc.ca/ english/research/papers/interprovincial/interprovincial- $h . h t m l$

« University Enrolment by Field of Study ». Statistique Canada. consultéle 06 mars 2006 dans : http:/ / www.statcan.ca/ Daily/ English/ 030331/d030331b.htm 
« Youth Electoral Participation - Survey and Analysis of Canadian Trends (October 2003) ». Élections Canada. consulté le 06 mars 2006 dans :

http:/ / www.elections.ca/ content.asp?section=med\&document=survey\&dir=eveyou/ forum\&l...

\section{Revues politiques spécialisées}

AARTS, Kees et Charlotte VAN HEES. « Lowering the Voting Age : European Debates and Experiences ». Electoral Insight, juillet 2003, pp.42-47.

ARCHER, Keith. « Increasing Youth Voter Registration : Best Practices in Targeting Young Electors ». Electoral Insight, juillet 2003, pp.26-30

BLAIS, André; GIDENGIL, Elisabeth; NADEAU, Richard; et NEVITTE, Neil. « Turned Off or Tuned Out? : Youth Participation in Politics ». Electoral Insight, juillet 2003, pp.9-14.

BLAIS, André; FOURNIER, Patrick; GIDENGIL, Elisabeth; NEVITTE, Neil; et RUBENSON, Daniel. «Accounting for the Age Gap in Turnout ». Acta Politica, 2004, volume 39, pp. 407-421

BLAIS, André; EVERITT, J oanna; FOURNIER, Patrick; GIDENGIL, Elisabeth; et NEVITTE, Neil. « Missing the Message : Young Adults and the Election Issues ». Electoral Insight, janvier 2005, 8 p.

BROWN, Wayne. « Electoral Facts ». Electoral Insight, janvier 2001, p.30.

BROWN, Wayne. « Rush the Vote ». Electoral Insight, juillet 2003, p.47.

FOURNIER, Bernard et Raymond HUDON. « How Old Is Old Enough to Vote? : Youth Participation in Society ». Electoral Insight, juillet 2003, pp.36-41.

HOWE, Paul. « Electoral Participation and the Knowledge Deficit ». Electoral Insight, juillet 2003, pp.20-25.

KINGSLEY, J ean-Pierre. « Youth Participation in Elections ». Electoral Insight, juillet 2005, pp.1-2.

O’NEIL, Brenda. « Examining Declining Electoral Turnout Among Canada's Youth ». Electoral Insight, juillet 2003, pp.15-19.

PAMMETT, J on H. « Youth in the Electoral Process ». Electoral Insight, janvier 2001, pp.14-17. TURCOTTE, André. « Different Strokes : Why Young Canadians Don't Vote ». Electoral Insight, janvier 2005, $7 \mathrm{p}$. 